\title{
An audit of calls to a specialist nurse-led rheumatology advice line related to antibiotic treatment and biological therapies
}

\author{
Joan Swan • Ursula Bond • Mortimer B. O'Connor • \\ Mark J. Phelan
}

Received: 7 February 2011/Accepted: 17 April 2013/Published online: 25 April 2013

(C) Springer-Verlag Berlin Heidelberg 2013

Dear Editor,

One of the primary goals of our nurse-led telephone advice line is to enhance and support self-management of our rheumatology patients. Over the recent years, evaluation of such nurse-led advice lines has been proven to be beneficial in such areas as promotion of self-management. One potential area in promotion of self-management is the area of biological therapies among inflammatory arthropathy patients.

Patients receiving biological therapies have been shown to have an increased risk of infections. In response to this, prior to commencing biological therapies, all our rheumatology patients are educated in relation to infections while prescribed biological therapies and also regarding deferral of biological therapy while receiving antibiotic treatments. An information pack, with relevant advice concerning individual biological therapy, is also given to patients. Those on intravenous (I.V.) biological infusions are advised to contact the nurse-led advice line if on antibiotic treatment during the week prior to infusion, to facilitate a potential IV biological therapy schedule adjustment. The aims of this education programme are to empower patients and promote self-management.

Recently, we conducted a retrospective audit of all calls received by our nurse-led advice line, in relation to antibiotic usage over a period of 6 months, was carried out (July-Dec 2009). Recorded data included the nature of call, the number of calls received, patients' demographics and source of call (patient, carer or primary care team). The

J. Swan · U. Bond · M. B. O'Connor $(\bowtie) \cdot$ M. J. Phelan Department of Rheumatology, South Infirmary Victoria University Hospital, Cork, Ireland

e-mail: mortimeroconnor@gmail.com primary objectives were to: (1) assess the calls received by the nurse-led advice line in relation to antibiotic treatment while on biological therapy and (2) promote patient confidence and encouragement to enhance self-management on biological therapies.

A total of 30 calls were received relating to antibiotic treatment while on biological therapies. Twenty-seven patient calls (90\%; 9 female, 18 male) and three general practitioner (GP) calls (all female patients) were received. Twenty-four calls ( $80 \%$ of all calls) related to subcutaneous biological therapies of which $12(50 \%)$ calls resulted in deferral of subcutaneous biologics for one dose only. Six calls concerned IV biological therapies, with no call resulted in rescheduling of IV biological therapy.

In conclusion, our nurse-led advice line has been shown to be beneficial in minimising missed biological therapies while receiving antibiotic therapy. The number of calls in relation to antibiotic treatment while on biological therapies was higher than expected. However, no follow-up call was received following the advice given. Following consultation with rheumatology nurses, rheumatologists and pharmacists, a decision has been made to develop an information leaflet, giving clear explanations on management, for all patients receiving antibiotics while on biological therapies. A guidance document published by the Royal College of Nursing supports this new approach [1].

\section{Reference}

1. Royal College of Nursing (2009) Assessing, managing and monitoring biologic therapies for inflammatory arthritis. RCN, London. ISBN 978-1-906633-13-4 